\title{
A Case of Metastatic Gastric Neuroendocrine Tumor: Therapeutic Considerations
}

\author{
Shin Young Kim In Sook Woo Ji Hyun Yang Chi Wha Han \\ Sang Young Roh Yun Hwa Jung \\ Division of Hematology-Oncology, Department of Internal Medicine, Yeouido St. Mary's \\ Hospital, College of Medicine, Catholic University of Korea, Seoul, Korea
}

\section{Key Words}

Stomach · Neuroendocrine tumor · Therapy

\begin{abstract}
Despite remarkable progression in the treatment and classification system of neuroendocrine tumor (NET), some questions have remained unanswered. The lack of an established treatment strategy for gastric NET is one of the problems. Because of its paucity, gastric NET is not discussed in independent, large-scaled prospective studies and tends to be excluded from clinical trials. Moreover, a separate classification system and some distinguished clinical features render the treatment of gastric NET more complicated. Here, we present a case of a female gastric NET patient with G2 proliferation index and multiple liver metastases. Based on the histologic grade and a high serum gastrin level, we initially treated her with somatostatin analogue. However, the patient did not respond. After that, cytotoxic chemotherapy with the etoposide plus cisplatin regimen only showed response in the short-term period. However, combination therapy with octreotide and interferon brought about significant regression of the tumor. Herein, we present our case together with a literature review of the treatment of metastatic gastric NET.

(c) 2014 S. Karger AG, Basel
\end{abstract}

\section{Introduction}

Along with the introduction of effective therapeutic agents such as somatostatin analogue (SSA), multityrosine kinase inhibitor and mammalian target of rapamycin inhibitor, much improvement has been made in the treatment of neuroendocrine tumors (NET). Especially, the PROMID [1] and RADIANT [2] studies proved the effectiveness of these new

Yun Hwa Jung, MD, Division of Hematology-Oncology

Department of Internal Medicine, Yeouido St. Mary's Hospital

College of Medicine, Catholic University of Korea

62, Yeouido-dong, Yeongdeungpo-gu, Seoul 150-713 (Korea)

E-Mail ksdmz@hanmail.net 
Kim et al.: A Case of Metastatic Gastric Neuroendocrine Tumor: Therapeutic Considerations

therapeutic agents in the treatment of mid gut and pancreatic NET, respectively. However, in the case of metastatic gastric NET, there is neither an established treatment strategy nor a prospective study because gastric NETs are not only rare but also mostly diagnosed in the early stage, which can be controlled by surgical resection. Therefore, gastric NET has been considered a part of the gastroenteropancreatic NET category and there have been no studies exclusively aimed at gastric NET. In the treatment of metastatic NET, a treatment approach according to the presence of hormonal symptom, embryological origin of primary site and pathologic grade is usually accepted as a principle. However, in the case of gastric NET, a separate treatment approach depending on a specific classification, which can be recognized based on clinical and histologic characteristics, is considered. In the classification of gastric NET, type I (74\% of the gastric NETs) is associated with chronic atrophic gastritis and hypergastrinemia, and type II (6\% of the gastric NETs) is associated with multiple endocrine neoplasia type I, Zollinger-Ellison syndrome and hypergastrinemia. Lastly, sporadic type III gastric neuroendocrine carcinoma (NEC; 13\% of the gastric NETs) is gastrin-independent and carries the worst prognosis.

In this paper, we describe the case of a female gastric NET patient with multiple liver metastases who was unresponsive to biologic therapy and cytotoxic chemotherapy. In addition, we look at the therapeutic strategy for metastatic gastric NET together with a literature review.

\section{Case Presentation}

A 75-year-old woman was referred to our hospital with epigastric pain. Upper endoscopic examination revealed a localized, fixed and firm subepithelial tumor (diameter, 2.2 $\mathrm{cm}$ ) in the peripyloric area, but there was no evidence of gastric ulcer in the entire stomach (fig. 1). After upper endoscopic examination, a stomach computed tomography (CT) was performed and revealed two non-enhancing, low-attenuated liver masses in S4 $(2.2 \mathrm{~cm})$ and S5 $(1.6 \mathrm{~cm}$ ) suggesting metastasis (fig. 2a). Sequentially performed liver MRI showed several additional metastatic nodules. Laparoscopic antrectomy with gastrojejunostomy and liver biopsy were performed, but hepatic metastatectomy was not tried due to multiplicity. The biopsy result of both gastric mass and metastatic hepatic nodule revealed a welldifferentiated NEC (NET G2 by 2010 WHO classification). Pathologically, the tumor was $37 \times$ $21 \mathrm{~mm}$ in size with a negative margin (distance from resected margin: $0.5 \mathrm{~cm}$ ) and infiltrated the muscular propria layer. Microscopically, the tumor cells were medium-sized and uniform in shape and showed moderate cellularity with mild mitosis $(<1 / 50$ per high-power field $)$ (fig. 3a). On immunohistochemical staining, the tumor cells were positive for chromogranin A and CD56a (fig. 3b, c). The Ki-67 labeling index was 4\% (fig. 3d), and the serum gastrin level was increased to $1,832 \mathrm{pg} / \mathrm{ml}$ (normal range 0-108). The vitamin B 12 level was 350 $\mathrm{pg} / \mathrm{ml}$ (normal range 211-911), chromogranin A level was $356.29 \mathrm{ng} / \mathrm{ml}$ (normal range 2794), 5-hydroxy indole acetic acid level was $3.04 \mathrm{mg} /$ day (normal range $<10$ ) and serotonin (5-hydroxytryptamin) was $48 \mathrm{ng} / \mathrm{ml}$ (normal range <200). However, CT and MRI did not localize any tumor assumed of gastrinoma. Considering the gastrin level and histologic grade, the patient could be classified into type I gastric NET. Moreover, she complained of abdominal pain with diarrhea and was therefore treated with long-acting repeatable (LAR) sandostatin at a dose of $20 \mathrm{mg}$ intramuscularly every 28 days. However, abdominal CT, which was performed 3 months later, revealed progression of the hepatic metastases without tumor recurrence in the operated bed (fig. 2b). The treatment was changed to cytotoxic chemotherapy. The patient received an etoposide plus cisplatin (EP) combination 
Kim et al.: A Case of Metastatic Gastric Neuroendocrine Tumor: Therapeutic Considerations

chemotherapy (etoposide $100 \mathrm{mg} / \mathrm{m}^{2}$ on days $1-3$, cisplatin $75 \mathrm{mg} / \mathrm{m}^{2}$ on day 1 ). Despite 3 cycles of EP chemotherapy, response evaluation identified a stable disease status, and eventually, her disease progressed after 6 cycles of EP chemotherapy (fig. 2c). Because of the patient's economic situation and lack of grounds, targeted agents such as sunitinib and everolimus were infeasible. Her performance status deteriorated and diarrhea persisted. Therefore, we chose a combination therapy with interferon- $\alpha$ (IFN) and sandostatin LAR. On response evaluation after 3 months of treatment, she showed favorable response with regression of liver metastasis (fig. 4).

\section{Discussion}

Since Oberndorfer first denominated the carcinoid tumor in 1907, there has been much improvement in the classification and treatment of NET. Especially the introduction of the new WHO pathologic classification system, which is based on the Ki-67 labeling index and a few landmark studies such as PROMID and RADIANT, provided a useful guide to the oncologists dealing with gastroenteropancreatic NET. Based on these studies, SSA is known to be effective in the treatment of midgut NETs, and cytotoxic chemotherapy such as the EP regimen is known to be effective in the treatment of foregut NETs, including pancreatic NETs. However, NET can arise in most organs of the body and can show a variety of clinical features according to its origin and the degree of differentiation. Moreover, in the case of NETs that originate from rare primary sites, standard treatment strategies have not been defined yet. Gastric NET is one of the unknown types of NETs. Although a division according to the embryological origin, which divides NETs into foregut, midgut and hindgut NETs, has been used in practice, it cannot guarantee the homogeneity between the different organs inside the same embryological category. That is to say, although gastric NET embryologically originates from the foregut, we do not know exactly whether it shows an aspect similar to NET of the pancreas. Moreover, gastric NET has a few distinctive features in its pathogenesis and natural course. Gastric NET is known to be derived from the enterochromaffin-like (ECL) cells, which produce histamine, express the gastrin receptor and regulate gastric acid secretion. Gastrin, which is released by G cells in the antrum of the stomach, duodenum and the pancreas, stimulate the release of histamine in ECL cells. Therefore, neoplastic changes in ECL cells are often associated with elevated serum gastrin levels [3]. Based on this unique pathogenetic background, a separate classification system for gastric NET was first defined by Rindi et al. [4] in 1993. According to this classification, gastric NET can be subdivided into types I, II and III, depending on the gastrin level and source of hypergastrinemia. A treatment strategy according to that system was also proposed [5]. Because of the relatively benign clinical course, low metastatic potential and low histologic grade of type I and II gastric NETs, the treatment strategy for these types of NETs was focused on local control, and the principal treatment was conservative, including endoscopic follow-up or endoscopic mucosal resection. Even in the case of multicentric tumor with liver metastasis, antrectomy and the consequent normalization of gastrin levels was considered sufficient for the control of the remnant lesion. As for medical treatment, biologic therapy such as SSA and IFN was considered for the treatment of type I and II gastric NETs. A few studies also demonstrated a therapeutic value of octreotide in NETs of gastroduodenal origin [6, 7]. Besides that, Khuroo et al. [8] showed that long-acting SSA injections reduced serum gastrin and serum chromogranin A levels with a 50\% reduction in the visible number of tumors in 3 patients with type I metastatic gastric carcinoids. IFN- $\alpha$ and $-\gamma$ have also been used in the treatment of gastrointestinal NETs, regardless of functionality. Immune-mediated cytotoxicity, inhibition 
Kim et al.: A Case of Metastatic Gastric Neuroendocrine Tumor: Therapeutic Considerations

of progression of the cell cycle from the $\mathrm{S}$ to the G2 phase and inhibition of tumor angiogenesis were proposed as main mechanisms of IFN [9]. However, systemic side effects such as flu-like symptoms and hypothyroidism hindered widespread usage as a first-line treatment. According to the European Neuroendocrine Tumor Society (ENETS) guidelines, SSA is known to be valuable for the subgroups of patients with slowly progressive and low proliferative NETs (G1) of gastroduodenal origin, and in cases of metastatic G3 NECs or welldifferentiated but rapidly progressive metastatic disease, combinations of etoposide and cisplatin are recommended [10]. However, it is not certain whether the histologic grade depending on the rate of cellular proliferation correlates with the classification based on gastrin levels and how to integrate the gastric NET and WHO classifications into the same treatment strategy for gastric NETs, especially in cases of metastatic disease. Moreover, there is the opinion that dysplastic cells are less dependent on gastrin stimulation and do not regress in response to reduced circulating gastrin levels. Indeed, a few metastatic cases with a bad prognosis have been reported in type I or II gastric NETs $[11,12]$.

In our case, the biopsy results showed a well-differentiated, G2 NEC with $4 \%$ of Ki-67 mitosis and an elevated gastrin level of $1,832 \mathrm{pg} / \mathrm{ml}$. However, there was no evidence of endocrine neoplasia at the other site. Based on the histologic grade and gastrin level, type I gastric NET was suspected. Therefore, we expected good response to SSA, but somatostatin did not achieve response and disease progression was faster than expected. This was the reason why we chose EP chemotherapy as a salvage treatment. However, after 6 cycles of EP chemotherapy, the tumor eventually progressed within only 2 months. Because the general condition of our patient was infeasible for another cytotoxic chemotherapy, we again used SSA for the relief of functional symptoms and stabilization of the tumor. In addition, we added IFN- $\alpha$ and obtained favorable response. According to previous studies comparing SSA or IFN alone with a combination of the two drugs, response was not superior in any of these groups, whereas the side effects leading to an interruption of the therapy were more frequent in the combination group [13]. However, according to Kolby et al. [14], the combination of IFN- $\alpha$ and SSA reduced tumor progression better and for a longer time than either agent alone and was well tolerated because SSA can reduce side effects of IFN- $\alpha$, although the study was aimed at midgut NET. One review article about the combination of IFN- $\alpha$ and SSA presented data showing an advantage of additional IFN- $\alpha$ after progression following SSA alone. However, there is not enough statistical evidence for an upfront use of the combination of IFN- $\alpha$ and SSA [15].

In summary, for the treatment of metastatic gastric NET, both the clinical classification according to the gastrin level and the pathologic grade should be considered. SSA can be considered a first-line medical treatment for type I and II metastatic gastric NET. However, the combination of SSA and INF- $\alpha$ can be an effective option in cases showing aggressive tumor behavior or after failure of SSA alone.

\section{Disclosure Statement}

There are no conflicts of interest relevant to this article. 


\section{Case Reports in Oncology}

\begin{tabular}{l|l}
\hline Case Rep Oncol 2014;7:266-272 \\
\hline DOI: $10.1159 / 000362516$ & $\begin{array}{l}\text { C 2014 S. Karger AG, Basel } \\
\text { www.karger.com/cro }\end{array}$ \\
\hline
\end{tabular}

Kim et al.: A Case of Metastatic Gastric Neuroendocrine Tumor: Therapeutic Considerations

\section{References}

1 Rinke A, Müller HH, Schade-Brittinger C, et al: Placebo-controlled, double-blind, prospective, randomized study on the effect of octreotide LAR in the control of tumor growth in patients with metastatic neuroendocrine midgut tumors: a report from the PROMID Study Group. J Clin Oncol 2009;27:4656-4663.

2 Yao JC, Shah MH, Ito T, et al: Everolimus for advanced pancreatic neuroendocrine tumors. N Engl J Med 2011;364:514-523.

-3 Burkitt MD, Pritchard DM: Review article: pathogenesis and management of gastric carcinoid tumours. Aliment Pharmacol Ther 2006;24:1305-1320.

4 Rindi G, Luinetti O, Cornaggia M, Capella C, Solcia E: Three subtypes of gastric argyrophil carcinoid and the gastric neuroendocrine carcinoma: a clinicopathologic study. Gastroenterology 1993;104:994-1006.

5 Gilligan CJ, Lawton GP, Tang LH, West AB, Modlin IM: Gastric carcinoid tumors: the biology and therapy of an enigmatic and controversial lesion. Am J Gastroenterol 1995;90:338-352.

6 Caplin ME, Hodgson HJ, Dhillon AP, et al: Multimodality treatment for gastric carcinoid tumor with liver metastases. Am J Gastroenterol 1998;93:1945-1948.

7 Tomassetti P, Migliori M, Caletti GC, et al: Treatment of type II gastric carcinoid tumors with somatostatin analogues. N Engl J Med 2000;343:551-554.

-8 Khuroo MS, Khuroo MS, Khuroo NS: Treatment of type I gastric neuroendocrine tumors with somatostatin analogs. J Gastroenterol Hepatol 2010;25:548-554.

-9 Oberg K: Chemotherapy and biotherapy in the treatment of neuroendocrine tumours. Ann Oncol 2001;12(suppl 2):S111-S114.

$\checkmark 10$ Delle Fave G, Kwekkeboom DJ, Van Custem E, et al: ENETS Consensus Guidelines for the management of patients with gastroduodenal neoplasms. Neuroendocrinology 2012;95:74-87.

$\checkmark 11$ Spampatti MP, Massironi S, Rossi RE, et al: Unusually aggressive type 1 gastric carcinoid: a case report with a review of the literature. Eur J Gastroenterol Hepatol 2012;24:589-593.

12 Bordi C, Falchetti A, Azzoni C, et al: Aggressive forms of gastric neuroendocrine tumors in multiple endocrine neoplasia type I. Am J Surg Pathol 1997;21:1075-1082.

13 Arnold R, Rinke A, Klose KJ, et al: Octreotide versus octreotide plus interferon-alpha in endocrine gastroenteropancreatic tumors: a randomized trial. Clin Gastroenterol Hepatol 2005;3:761-771.

$>14$ Kolby L, Persson G, Franzen S, et al: Randomized clinical trial of the effect of interferon alpha on survival in patients with disseminated midgut carcinoid tumours. Br J Surg 2003;90:687-693.

15 Fazio N, De Braud F, Delle Fave G, Oberg K: Interferon-alpha and somatostatin analog in patients with gastroenteropancreatic neuroendocrine carcinoma: single agent or combination? Ann Oncol 2007;18:1319.

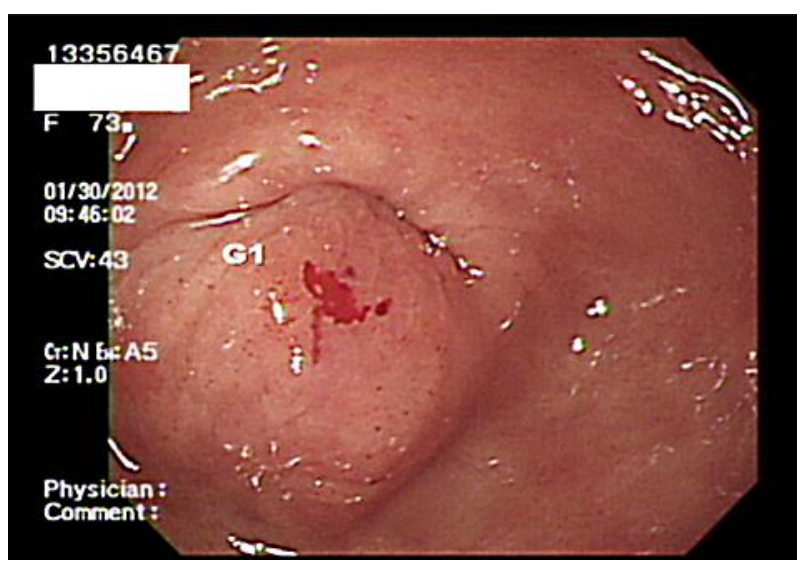

Fig. 1. Upper endoscopic examination revealed a localized, fixed and firm subepithelial tumor (diameter, $2.2 \mathrm{~cm}$ ) in the peripyloric area. 


\section{Case Reports in Oncology}
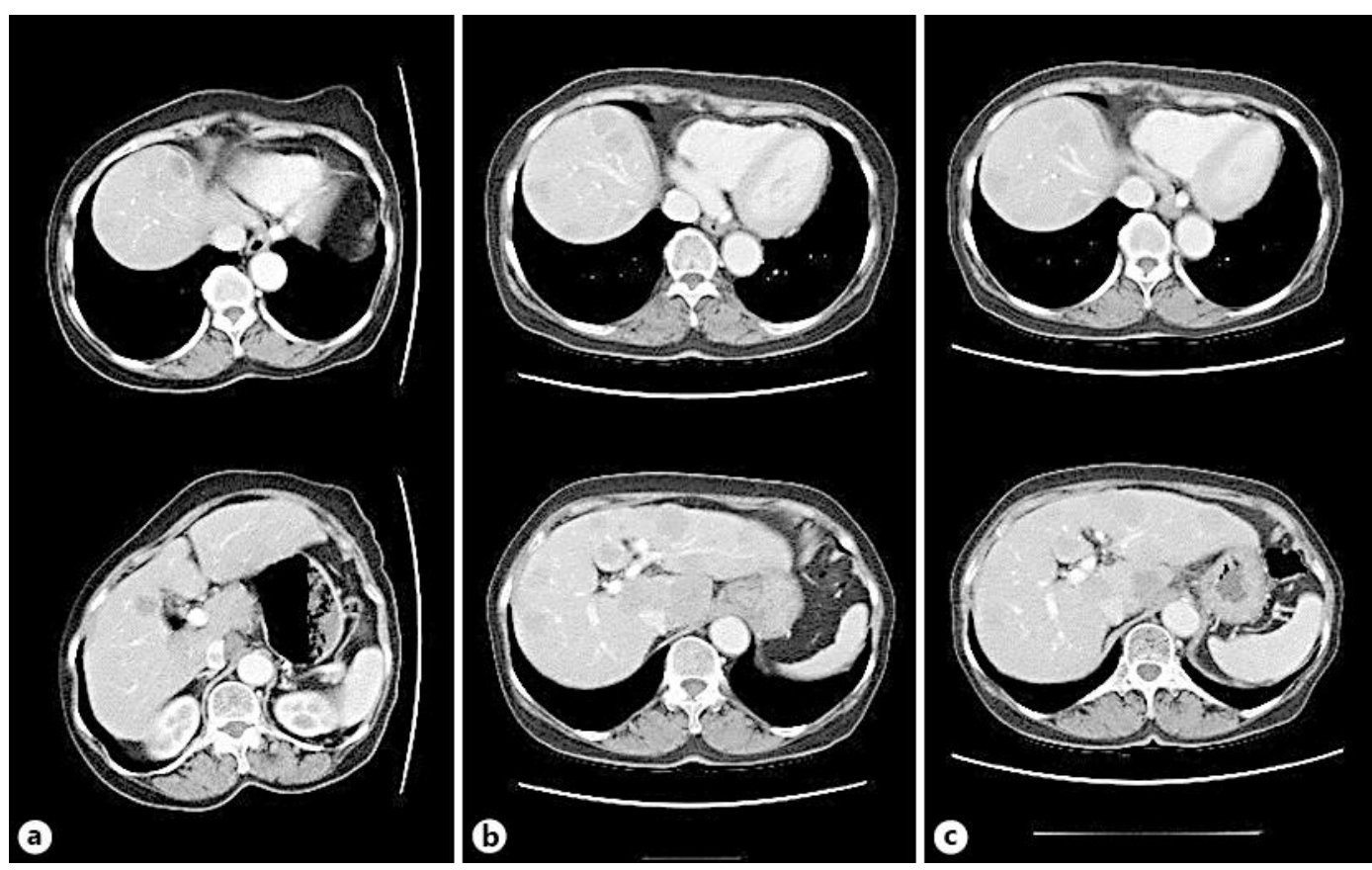

Fig. 2. a A Stomach CT scan before the treatment revealed two non-enhancing, low-attenuated liver masses in S4 $(2.2 \mathrm{~cm})$ and S5 $(1.6 \mathrm{~cm})$ suggesting metastasis. b Abdominal CT scan 3 months after sandostatin LAR therapy. There was progression of hepatic metastasis without tumor recurrence in the operated bed. c Follow-up abdominal CT after 6 cycles of EP chemotherapy. There was no significant interval change in the size and number of hepatic metastasis. 


\section{Case Reports in Oncology}

Case Rep Oncol 2014;7:266-272
DOI: $10.1159 / 000362516$

Kim et al.: A Case of Metastatic Gastric Neuroendocrine Tumor: Therapeutic Considerations
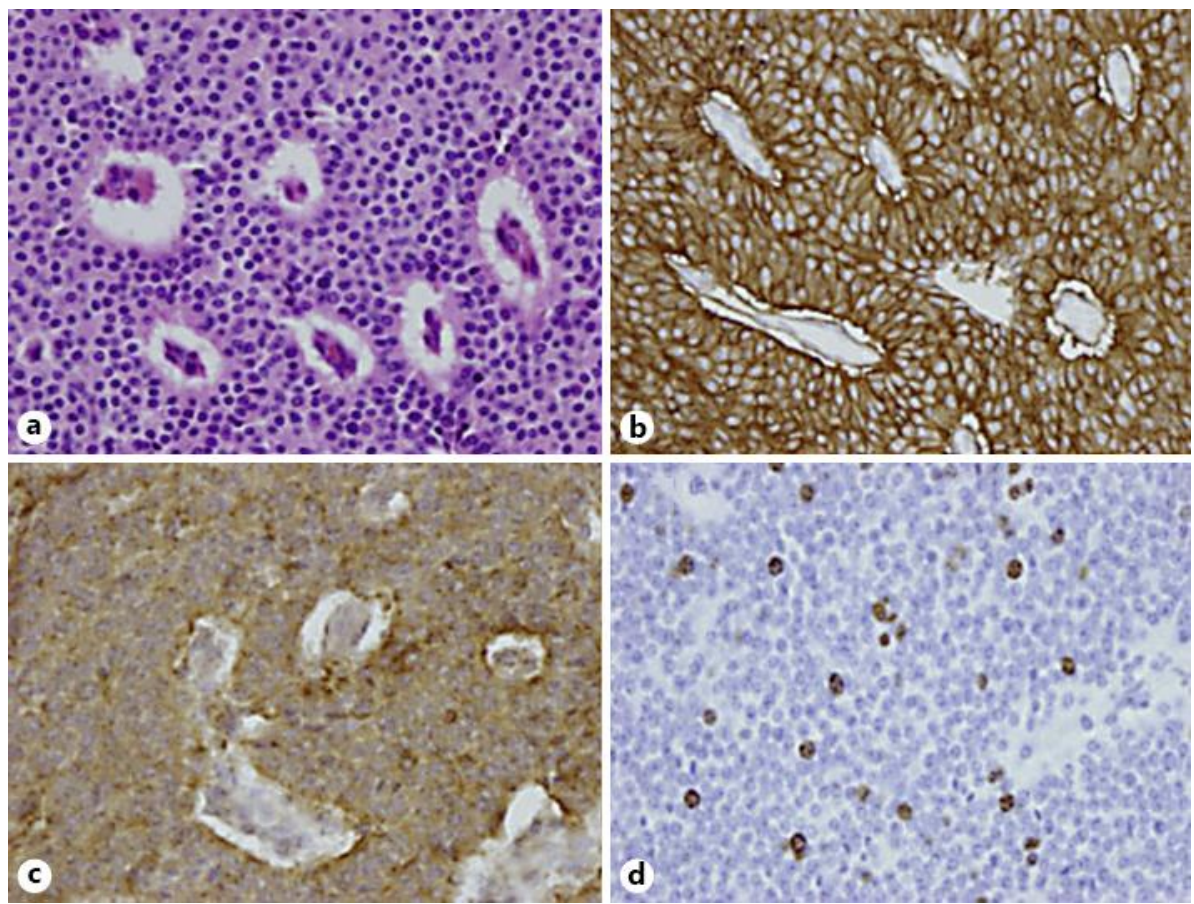

Fig. 3. a Histology. The tumor was composed of medium-sized, round or polygonal cells with eosinophilic cytoplasm and round nuclei with a fine chromatin pattern also known as 'salt-and-pepper nuclei'. HE. $\times 400$. b, c Immunohistochemistry. The tumor cells exhibited positive staining for chromogranin A and CD56. $\mathbf{d}$ The Ki-67 labeling index was $4 \%$.
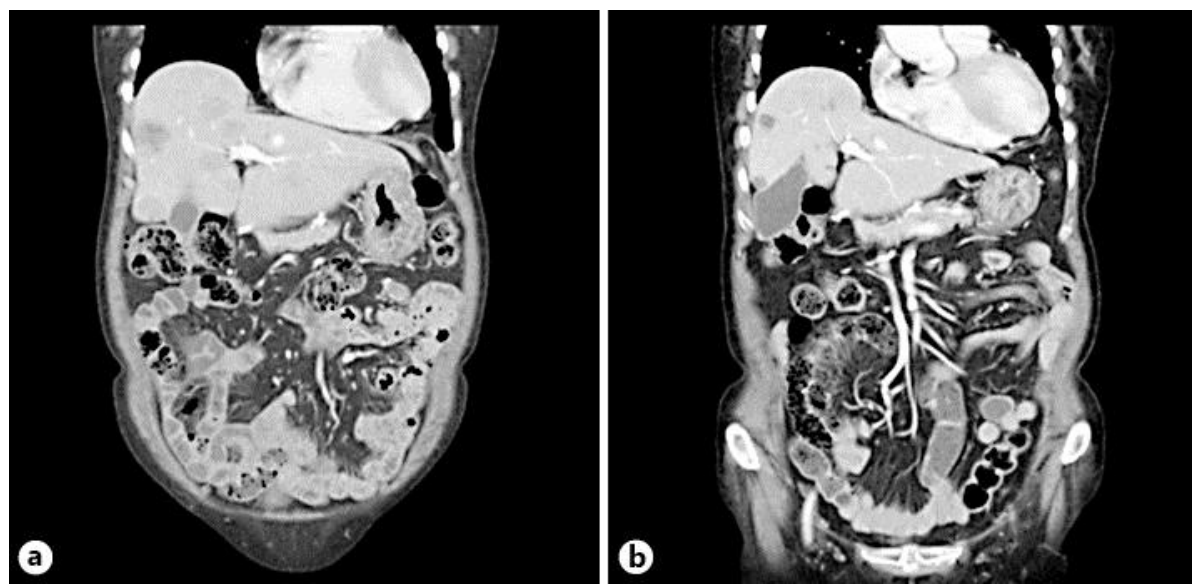

Fig. 4. The stomach CT (b) performed after 3 months of octreotide plus IFN- $\alpha$ showed an interval decrement of metastatic liver masses compared to the previous CT scan (a). 